\title{
THE IMPACT OF ANTITUBERCULOSIS DRUG-INDUCED HEPATOTOXICITY TO SUCCESFUL TUBERCULOSIS TREATMENT IN INDONESIA
}

\author{
NISA MARIA ${ }^{1 *}$, MAKSUM RADJI $^{1}$, ERLINA BURHAN ${ }^{2,3}$ \\ ${ }^{1}$ Department of Pharmacy, Faculty of Pharmacy, University of Indonesia, Depok, Indonesia. ${ }^{2}$ Department of Pulmonology and Respiratory \\ Medicine, Faculty of Medicine, University of Indonesia, Jakarta, Indonesia. ${ }^{3}$ Department of Pulmonology and Respiratory, Persahabatan \\ Hospital, Jakarta, Indonesia. Email: nisamaria88@gmail.com
}

Received: 01 June 2017, Revised and Accepted: 27 July 2017

ABSTRACT

Objective: This study aimed to evaluate the impact of antituberculosis (anti-TB) drug-induced hepatotoxicity (DIH) to outcome TB treatment.

Methods: A cohort retrospective study conducted at a tertiary hospital in Jakarta - Indonesia, from the period of 2013-2016. A total of 76 samples of TB patient with and without anti-TB DIH were analyzed.

Results: Successful outcome TB treatment for TB patient with anti-TB DIH is 47.4\% compared to TB patient without anti-TB DIH is $78.9 \%$. Relative risk (RR) analysis showed that risk of unsuccessful TB treatment for TB patient with anti-TB DIH is 2.50 fold higher (95\% confidence interval: 1.259 4.960) than TB patient without anti-TB DIH. Age, sex, and comorbidities are not statistically significant to outcome TB treatment. For TB patient with anti-TB DIH, onset anti-TB DIH and recurrence anti-TB DIH also not statistically significantly influence outcome TB. The mean duration of treatment for a successful outcome for TB patient with and without anti-TB DIH was statistically significant ( $<<0.05)$, respectively, $8.44 \pm 1.85$ and $6.52 \pm 0.93$ months.

Conclusion: Anti-TB DIH increases the risk of unsuccessful and prolonged duration TB treatment.

Keywords: Antituberculosis, Drug-induced hepatotoxicity, Outcome.

(C) 2017 The Authors. Published by Innovare Academic Sciences Pvt Ltd. This is an open access article under the CC BY license (http://creativecommons. org/licenses/by/4. 0/) DOI: http://dx.doi.org/10.22159/ajpcr.2017.v10i11.20386

\section{INTRODUCTION}

Tuberculosis (TB) is one of the most common infectious diseases that caused by Mycobacterium tuberculosis [1]. Based on the World Health Organization (WHO) TB Reports, globally in 2014, there were an estimated 9.6 million incident cases of TB. The national prevalence TB survey in Indonesia estimated that there are about 1 million new TB cases. In the major implication of survey results, Indonesia becomes the second largest number of TB cases (10\% of the global total), and TB warrant being one of the top health priorities in Indonesia [2].

Anti-tuberculosis (anti-TB) drugs can kill M. tuberculosis effectively but also known can cause adverse drug reactions (ADRs) $[3,4]$. The study in India showed that common ADR observed from first-line anti-TB drugs was disturbed liver transaminases (33.33\%), followed by nausea and vomiting (28.88\%). Other ADRs also included hepatitis, headache, rash, constipation, fever, flu-like syndrome, blurred vision and optic neuritis, metabolic disturbances including hyperglycemia, and diarrhea [5]. Other study showed that the percentage of patients with single and more than one ADR from first-line anti-TB drugs was $24.09 \%$ and $75.9 \%$, respectively [6].

The four combination first-line anti-TB drugs are isoniazid $(\mathrm{H})$, rifampicin (R), pyrazinamide $(\mathrm{Z})$, and ethambutol $(\mathrm{E})$, and the first three of the drugs have a potential to cause drug-induced hepatotoxicity (DIH) $[4,7]$. The incidence of DIH due to anti-TB standard reported varied between $2.0 \%$ and $28.0 \%$ depending on population differences and the definition of DIH [6]. DIH diminishes the effectiveness of anti-TB treatment, may cause non-adherence, and can cause treatment failure, recurrence, or drug resistance [8]. DIH is responsible for significant morbidity and mortality of the TB patient if these drugs continued after symptoms of hepatotoxicity develop [7].

The management therapy for TB patients with anti-TB DIH is important to ensure the successful TB treatment and not recurrence
DIH. This research aimed to evaluate the occurred anti-TB DIH from TB patients that used first-line anti-TB drugs, clinical features of anti-TB DIH, and impact anti-TB DIH to TB treatment and outcome TB treatment.

\section{METHODS}

\section{Study design}

In this cohort retrospective study, using inpatient and outpatient medical record TB patients, data collected from medical record TB patient with initial TB treatment on January 2013-September 2016 at Persahabatan Hospital, a tertiary referral hospital in Jakarta, Indonesia. A total sampling applied for medical records of TB patient with DIH, while for medical records, TB patients without DIH using systematic random sampling. Protocol of this study was approved by the Ethics Committee of the Faculty of Medicine, University of Indonesia and also from Hospital Ethics Committee.

\section{Inclusion criteria}

New case pulmonary TB patients that used the combination of first-line anti-TB drugs (isoniazid, rifampicin, pyrazinamide, and ethambutol), both male and female patient with age 18-65 years, and no symptoms of hepatitis at the initial TB treatment that recorded at medical record patient were enrolled in this study.

\section{Exclusion criteria}

TB patients with human immunodeficiency virus infection or history of alcohol consumption for at least 1 year or history of chronic diarrhea were excluded from this study. Patients with a concomitant administration with other hepatotoxic drugs or use herbal medications and patient with serological evidence of viral hepatitis (A, B, C, or E) also excluded from this study [7].

\section{Diagnostic criteria}

The criteria for diagnosed DIH due to anti-TB drugs refer to the National 
Guideline for Tuberculosis in Indonesia. Patient diagnosed DIH if the patient met at least one of the following criteria [9]:

1. Jaundice and symptoms of nausea and vomiting were present;

2. Symptoms were present and enzyme aspartate transaminase (AST), alanine transaminase (ALT) $\geq 3 \times$ upper limit normal (ULN);

3. No clinical symptoms, total bilirubin (TBil) $>2 \times$ ULN

4. No clinical symptoms, AST, ALT $\geq 5 \times$ ULN

The severity of hepatotoxicity classified according to the WHO Toxicity Classification Standards divided into three grades $[4,10]$ :

1. Mild defined as serum ALT level is 2-5 times of ULN and normal TBil level;

2. Moderate defined as serum ALT level is 5-10 times of ULN or serum ALT, or AST level is $<5$ times of ULN, and TBil level is 2-5 times of ULN;

3. Severe defined as both ALT or AST and TBil level is more than 5 times of ULN, or TBil level is more than 2 times of ULN with ascites and/or encephalopathy or other organ failures.

\section{Outcome TB}

Outcome TB treatment recorded based on the WHO classification [11]. In this study, TB outcome treatment divided into two categories, i.e., successful and unsuccessful outcome. TB patient categorized as a successful outcome if patient cured and treatment completed. Treatment failure, patient death, lost to follow-up, and not evaluated/ transfer out categorized as an unsuccessful outcome [8]

\section{Statistic analysis}

SPSS version 21.0 used for data analysis with $95 \%$ confidence interval (CI). The $\mathrm{p}<0.05$ considered have statistically significant. Mann-Whitney $\mathrm{U}$ test used for continuous variables. Chi-square and Fisher's exact test used to compare categorical variables. Anti-TB DIH was the dependent variable and outcome TB was the independent variable, whereas age, sex, and concomitant diseases were the confounding variable. The potential impact of anti-TB DIH to the TB outcome analyzed with a RR.

\section{RESULTS}

A total of 76 samples that met the inclusion and exclusion criteria were analyzed. Patients demographic showed in Table 1. All patients received anti-TB based on patient's body weight. Sex, age, smear sputum at initial treatment, and comorbidities are not statistically significant ( $p>0.05)$ influence on the occurrence of anti-TB DIH.

\section{Clinical features of anti-TB DIH}

The earlier onset of anti-TB DIH was 3 days, and the latest was 87 days from the initial of consumption anti-TB drugs. The number of patient with onset anti-TB DIH within 1 week is $23.7 \%$, onset anti-TB DIH $>1$ week until 30 days is $63.2 \%$, and $13.2 \%$ occurred more than 30 days after initial treatment with anti-TB drugs. The mean onset of anti-TB DIH was $19.95 \pm 17.14$ days. The onset anti-TB DIH in this study showed that most of the anti-TB DIH occurred in the first 30 days of TB treatment (86.9\%). For all the 38 anti-TB DIH cases, 33 patients (86.8\%) hospitalized.

The liver function test has been conducted for TB patient at the beginning of diagnosed DIH is AST, ALT, and Tbil. The mean of test result are $282 \pm 248 \mathrm{U} / \mathrm{L}$ (reference value $0-37 \mathrm{U} / \mathrm{L}$ ); $188 \pm 180 \mathrm{U} / \mathrm{L}$ (reference value $0-40 \mathrm{U} / \mathrm{L}$ ); and $2.9 \pm 3.2 \mathrm{mg} / \mathrm{dL}$ (reference value 0.1 $1.1 \mathrm{mg} / \mathrm{dL}$ ). Mann-Whitney U test shown that mean of AST, ALT, and Tbil not statistically significant for onset anti-TB DIH within 7 days and $>7$ days. Anorexia occurs for most of TB patient with anti-TB DIH (97.4\%). The other clinical symptoms related to anti-TB DIH that occurs are nausea $(73.7 \%)$, vomiting $(55.3 \%)$, and jaundice $(15.8 \%)$.

In terms of anti-TB DIH severity, 16 patients (44.7\%) had mild hepatotoxicity, 17 patients (44.7\%) had moderate hepatotoxicity, and five patients (13.2\%) had severe hepatotoxicity. TB patient with onset anti-TB DIH within 7 days after initial treatment mostly had mild
Table 1: Data demographic patients

\begin{tabular}{|c|c|c|c|}
\hline \multirow[t]{2}{*}{ Characteristics } & \multicolumn{2}{|l|}{$\mathrm{N}=38(\%)$} & \multirow{2}{*}{$\begin{array}{l}\text { p value } \\
\text { Chi-square }\end{array}$} \\
\hline & $\begin{array}{l}\text { TB patient } \\
\text { with } \\
\text { anti-TB DIH }\end{array}$ & $\begin{array}{l}\text { TB patient } \\
\text { without } \\
\text { anti-TB DIH }\end{array}$ & \\
\hline \multicolumn{4}{|l|}{ Sex } \\
\hline Male & $19(50.0)$ & $23(60.5)$ & 0.356 \\
\hline Female & $19(50.0)$ & $15(39.5)$ & \\
\hline \multicolumn{4}{|l|}{ Age (years) } \\
\hline $18-\leq 50$ & $25(65.8)$ & 28 (73.7) & 0.454 \\
\hline$>50-65$ & $13(34.2)$ & $10(26.3)$ & \\
\hline Mean age (year) \pm SD & $40.29 \pm 14.47$ & $38.39 \pm 12.89$ & \\
\hline \multicolumn{4}{|l|}{$\begin{array}{l}\text { Smear sputum at initial } \\
\text { treatment }\end{array}$} \\
\hline Positive & 27 (71.1) & $31(81.6)$ & 0.622 \\
\hline Negative & $11(28.9)$ & $7(18.4)$ & \\
\hline \multicolumn{4}{|l|}{ Comorbidities } \\
\hline Yes & 28 (73.7) & 28 (73.7) & 1.000 \\
\hline No & $10(26.3)$ & $10(26.3)$ & \\
\hline
\end{tabular}

Table 2: Clinical features of TB patient with anti-TB DIH

\begin{tabular}{lll}
\hline Clinical features & $\begin{array}{l}\text { TB patient } \\
\text { with anti-TB } \\
\text { DIH (N=38) }\end{array}$ & $\begin{array}{l}\text { Reference } \\
\text { value }\end{array}$ \\
\hline $\begin{array}{ll}\text { Onset of anti-TB DIH } \\
\text { Min-max (days) }\end{array}$ & $3-87$ & \\
$\quad$ Mean (days) \pm SD & $19.95 \pm 17.14$ & \\
Laboratory findings & & \\
AST (U/L) & $282 \pm 248$ & $0-37$ \\
ALT (U/L) & $188 \pm 180$ & $0-40$ \\
TBil (mg/dL) & $2.9 \pm 3.2$ & $0.1-1.1$ \\
Clinical symptoms (\%) & & \\
Anorexia & $37(97.4)$ & \\
Nausea & $28(73.7)$ & \\
Vomiting & $21(55.3)$ & \\
Jaundice & $6(15.8)$ & \\
Requirement of hospitalization (\%) & $33(86.8)$ & \\
Severity DIH & & \\
Mild & $16(42.1)$ & \\
Moderate & $17(44.7)$ & \\
Severe & $5(13.2)$ & \\
\hline
\end{tabular}

TB: Tuberculosis, DIH: Drug-induced hepatotoxicity, AST: Aspartate transaminase, ALT: Alanine transaminase, TBil: Total bilirubin, SD: Standard deviation

Table 3: Impact of anti-TB DIH on TB treatment

\begin{tabular}{ll}
\hline Impact of anti-TB DIH & $\begin{array}{l}\text { TB patient with anti-TB DIH } \\
\mathbf{N}=\mathbf{3 8}(\%)\end{array}$ \\
\hline $\begin{array}{l}\text { Management after anti-TB DIH } \\
\text { Interruption (stop all anti-TB }\end{array}$ & $30(78.9)$ \\
drugs) & $6(15.8)$ \\
Substitution therapy & $2(5.3)$ \\
No change/treatment continue & \\
Anti-TB drugs after anti-TB DIH & \\
HRES & $20(57.2)$ \\
HRZE & $11(31.4)$ \\
Other combinations & $4(11.4)$ \\
Recurrence anti-TB DIH & $4(11.4)$ \\
No. of patients (\%) & \\
Mean (days) \pm SD &
\end{tabular}

${ }^{a}$ Total $=35$ patients. ${ }^{\mathrm{b}}$ Time recurrence of anti-TB DIH assessed from day firs reintroduced anti-TB drugs until recurrence anti-TB DIH. TB: Tuberculosis, DIH: Drug-induced hepatotoxicity, SD: Standard deviation

hepatotoxicity (66.7\%). TB patient with onset anti-TB DIH $>7$ days mostly had moderate hepatotoxicity (51.7\%). 
Impact of anti-TB DIH on TB treatment

The hepatotoxic anti-TB drugs discontinued after DIH present for most of the patient in this study (94.7\%). For $78.9 \%$ of patients, all anti-TB drugs stopped after diagnosed anti-TB DIH, and the others $15.8 \%$ of patients were given substitution therapy with ethambutol and streptomycin after discontinued hepatotoxic anti-TB drugs. This study also found that $5.3 \%$ was continued TB treatment while anti-TB DIH develops with the same regimen therapy, i.e., HRZE.

Reintroduced of anti-TB drugs for TB patient who stopped all anti-TB drugs after anti-TB DIH present started when liver function test and clinical symptoms of DIH was improved. The duration time from onset of anti-TB DIH until reintroduced was $11.90 \pm 6.72$ days (2-28 days). For TB patients with anti-TB DIH that received substitution therapy after anti-TB DIH present, the duration until starting titration of isoniazid

Table 4: Correlation severity of anti-TB DIH with hepatoprotective agent

\begin{tabular}{llll}
\hline Severity of anti-TB DIH & \multicolumn{3}{l}{ Hepatoprotective agent } \\
\cline { 2 - 4 } & $\begin{array}{l}\text { One } \\
\text { drug (\%) }\end{array}$ & $\begin{array}{l}\text { Two } \\
\text { drugs (\%) }\end{array}$ & $\begin{array}{l}\text { Three } \\
\text { drugs (\%) }\end{array}$ \\
\hline Mild & $4(66.6)$ & $12(38.7)$ & 0 \\
Moderate & $1(16.7)$ & $15(48.4)$ & $1(100)$ \\
Severe & $1(16.7)$ & $4(12.9)$ & 0 \\
Total & $6(100)$ & $31(100)$ & $1(100)$ \\
\hline
\end{tabular}

TB: Tuberculosis, DIH: Drug-induced hepatotoxicity

Table 5: Outcome TB treatment

\begin{tabular}{lll}
\hline Outcome & $\mathbf{N = 3 8}(\%)$ & \\
\cline { 2 - 3 } & $\begin{array}{l}\text { TB patient with } \\
\text { anti-TB DIH }\end{array}$ & $\begin{array}{l}\text { TB patient without } \\
\text { anti-TB DIH }\end{array}$ \\
\hline $\begin{array}{ll}\text { Successful } \\
\text { Cure }\end{array}$ & $5(13.2)$ & $19(50.0)$ \\
$\quad$ Treatment completed & $13(34.2)$ & $11(28.9)$ \\
Total & $18(47.4)$ & $30(78.9)$ \\
Unsuccessful & & \\
Treatment failure & 0 & 0 \\
Death & $5(13.2)$ & 0 \\
Lost to follow-up & $13(34.2)$ & $4(10.5)$ \\
$\quad$ Not evaluated & $2(5.3)$ & $4(10.5)$ \\
Total & $20(52.6)$ & $8(21.1)$ \\
Total & $38(100)$ & $38(100)$ \\
\hline
\end{tabular}

TB: Tuberculosis, DIH: Drug-induced hepatotoxicity and rifampicin was 7.2 \pm 2.9 days (4-14 days) after the administration of ethambutol and streptomycin. The combination of anti-TB drugs for intensive phase after anti-TB DIH is HRES for 20 patients (57.2\%); HRZE for 11 patients (31.4\%); and others combination for four patients (11.4\%). Three (3) patients were deaths before the reintroduced started or an ongoing process of anti-TB drugs titration.

The recurrence anti-TB DIH occurred in $4 / 35$ patients (11.4\%). The mean time of recurrence anti-TB DIH was 23.25 days (14-46 days) after anti-TB reintroduced. All the patients with recurrence anti-TB DIH showed the same clinical symptoms of hepatitis with first antiTB DIH. The clinical symptoms that occurred are anorexia, nausea, and vomiting. Two patients (18.2\%; $2 / 11$ patients) who recurrence DIH found from a group of patient that reintroduced combination of HRZE with full dosage from day first and other two TB patients $(10.0 \%$; $2 / 20$ patients) from a group that reintroduced with HRES. Recurrence anti-TB DIH occurred for all severity of DIH, one patient (25\%) with mild hepatotoxicity, two patients (50\%) with moderate hepatotoxicity, and one patient $(25 \%)$ with severe hepatotoxicity.

\section{Hepatoprotective agent}

The hepatoprotective agent has given to the TB patient with anti-TB DIH to improve the liver function. In this study, TB patient received hepatoprotective agent after DIH diagnosed, none of the TB patients received hepatoprotective agent for preventive. Most of TB patient with anti-TB DIH received combination two drugs hepatoprotective (81.6\%). The patient who received one hepatoprotective drug is $15.8 \%$, and patient who received combination three drugs hepatoprotective is $2.6 \%$. Combination two drugs have given for patient with mild hepatotoxicity $(38.7 \%)$ and TB patient with moderate hepatotoxicity $(48.8 \%)$. The one hepatoprotective drug has given to $66.6 \%$ of TB patients with mild hepatotoxicity as shown in Table 4.

\section{Outcome TB treatment}

The successful outcome for TB patient with anti-TB DIH (47.4\%) lower than TB patient without anti-TB DIH (78.9\%). TB patient with anti-TB DIH that outcome was unsuccessful (52.6\%) higher than TB patient without anti-TB DIH (21.1\%). Statistic analyzed showed that outcome TB between TB patient with and without anti-TB DIH is a statistically significant $(\mathrm{p}=0.004)$.

The mean duration of TB treatment for successful outcome between TB patient with and without anti-TB DIH was a statistically significant $(\mathrm{p}=0.000)$. A successful outcome TB patient with anti-TB DIH received more than 6 months of treatment for $83.8 \%$ (15/18 patients), while $70.0 \%$ (21/30 patients) of TB patient without anti-TB DIH received treatmentfor 6 months. The mean duration of TB treatment for the patient

Table 6: Duration TB treatment for successful outcome

\begin{tabular}{lll}
\hline Duration TB treatment for successful outcome & Patient with anti-TB DIH* & Patients without anti-TB DIH \\
& & $\begin{array}{l}\text { p } \\
\text { Mann-Whitney U }\end{array}$ \\
\hline 6 months (\%) & $3(16.7)$ & $9(70.0)$ \\
>6 months (\%) & $15(83.8)$ & 30.000 \\
Total (\%) & $18(100)$ & $6.52 \pm 0.93$ \\
Mean (months) \pm SD & $8.44 \pm 1.85$ & $6-9$ \\
Min-max (months) & $6-14$ & 1000 \\
\hline
\end{tabular}

*The duration TB of successful treatment calculated from day first of full combinations and dosage anti-TB drugs reintroduced for patient with anti-TB DIH.

TB: Tuberculosis, DIH: Drug-induced hepatotoxicity, SD: Standard deviation

Table 7: Relative risk for outcome anti-TB treatments between TB patient with and without anti-TB DIH

\begin{tabular}{lllll}
\hline Outcome TB & Results & & & \\
\cline { 2 - 5 } & With anti-TB DIH & Without anti-TB DIH & RR (95\% CI) & p value \\
\hline Unsuccessful outcome (\%) & $20(52.6)$ & $8(21.1)$ & $2.50(1.259-4.960)$ & 0.004 \\
Succesfful outcome (\%) & $18(47.4)$ & $30(78.9)$ & & \\
\hline
\end{tabular}

TB: Tuberculosis, DIH: Drug-induced hepatotoxicity, CI: Confidence interval, RR: Relative risk 
who experienced anti-TB DIH is $8.44 \pm 1.85$ months (6-14 months) while for the patient without anti-TB DIH is $6.52 \pm 0.93$ months (6-9 months). The duration of successful TB treatment calculated from initial TB treatment for TB patient without anti-TB DIH. For TB patient with antiTB DIH, the duration of successful TB treatment calculated from day first of full combinations and dosage anti-TB drugs reintroduced.

Lost to follow-up, TB patients with anti-TB DIH are three times (34.2\%) higher than TB patient without anti-TB DIH (10.5\%). The mean of duration from initial TB treatment until lost to follow-up for the patient with anti-TB DIH is longer (5.00 1.90 months) than the patient without anti-TB DIH (3.50 \pm 1.29 months) but not statistically significant ( $p>0.05)$.

RR analysis confirmed that anti-TB DIH increased the risk of unsuccessful TB treatment 2.50 fold (95\% CI: 1.259-4.960) higher than TB patient without anti-TB DIH. The prospective study conducted by Penghui et al. also had the similar result with this study [7].

Age, sex, and comorbidities were not statistically significant $(p>0.05)$ influenced the outcome TB treatment. Especially for TB patient with DIH, statistical analysis showed that onset of anti-TB DIH (cut point 7 and 30 days) and recurrent anti-TB DIH not statistically significant influence the outcome TB treatment for TB patients with anti-TB DIH ( $p>0.05$ ).

\section{DISCUSSION}

Analysis risk factor of anti-TB DIH showed that age and sex not statistically significant influence to occurrence anti-TB DIH. This result was similar to several studies which showed that there was no relationship between age and sex with anti-TB DIH [12-14]. Other study had a different result which showed that sex did not affect the increase in ALT level but age had an effect on the increase in ALT level in TB patients who were treated with first-line anti-TB drugs [15].

The liver function test is not a routine program that conducted for all TB patients [12]. In this retrospective study, liver function test performed after TB patient showed clinical symptoms related to DIH. After patient diagnosed had anti-TB DIH, all patients received hepatoprotective agent to improve the liver function. The result of liver function TB patient with DIH showed improvement within 1 week after onset of DIH. Another prospective study also found that serum transaminase patient with DIH returns to a normal value within 1-2 weeks after the TB drugs were discontinued [13]

The management of DIH for TB patient in this study refers to the publish recommendation from the British Thoracic Society [16], American Thoracic Society [17], WHO [11], and National Guidelines for Tuberculosis in Indonesia [9]. In general, the recommendation from guidelines is discontinued the hepatotoxic anti-TB drugs (HRZ) if AST or ALT is more than five times the ULN (with or without symptoms) or more than three times the ULN with jaundice and or hepatitis symptoms. In this study, hepatotoxic anti-TB drugs discontinued for most of TB patient with anti-TB DIH (94.7\%). The remaining 5.3\% of patients who continue therapy with the same regimen after DIH occurred are patients with an elevation of AST and ALT not reached 5 times the ULN with mild hepatotoxicity and onset DIH within 30 days after initial TB drugs.

Recurrence of anti-TB DIH occurred higher for patients who reintroduced combinations HRZE with full dosage from day first. The results of the present study were similar with study of Sharma et al. that found an incident recurrence of DIH was $10.9 \%$, and the highest incident was a group that reintroduced HRZ maximum dose from day first [18].

The prolonged TB treatment due to interruption of treatment for a patient with anti-TB DIH could cause a large number of lost to followup patient from TB treatment program. Clinical symptoms that experienced by the patient (nausea, vomiting, and anorexia) may also affect to patient compliance. The improvement of clinical symptoms or the patient's condition become worst also influences the decision of TB patient to continue the treatment.

Educating the patient to compliance with the TB treatment program can be reduced the number of lost to follow-up TB patient. For TB patient who experienced DIH, the education should emphasize the prolonged of the duration TB treatment. TB treatment for TB patients who experienced DIH can prolong for up to 18-24 months [11].

Anti-TB DIH encompasses a wide spectrum of liver injury ranging from the asymptomatic minimal elevation of liver enzymes to acute liver failure, often leading to death or liver transplantation [19]. Five patients $(13.2 \%)$ with anti-TB DIH death during TB treatment, one of them was diagnosed acute liver failure due to anti-TB DIH. The mortality rate in this study was similar to the study that conducted by Agal et al. with mortality rate of $16.6 \%$ [20]. The other prospective study showed that none of TB patients with DIH was death from group TB patient that performed routine monitoring liver function. The number of death was higher in the group that not perform routine liver functions test [21].

Age, sex, and comorbidities (diabetes mellitus with or without hypertension) not statistically significant influence the outcome TB treatment. This result is difference with other studies. Study conducted by Sellart et al. show that age $>55$ years and male sex are independent risk factors for poor outcome TB treatment [22]. Other study results show that diabetes mellitus increases the risk of failure TB treatment and death during the TB treatment [23].

Early detection of DIH is clinically important for several reasons. First, the early identification of DIH can minimize the duration of interruption of TB treatment and improvement of the liver function becomes faster due to minimal damage of the liver. Second, rapid intervention for TB patients with DIH (before jaundice occurred) may associate with good prognosis [24]. The results of this study showed that $5.3 \%$ of TB patient with anti-TB DIH can continue the TB treatment (not interrupted) using the same regimen. The onset anti-TB DIH of this patient is within 30 days after initial TB treatment with mild hepatotoxicity and no need for hospitalization.

\section{CONCLUSIONS}

Anti-TB DIH mostly occurs within 30 days after initial TB treatment (86.9\%) with the mean of onset is $19.95 \pm 17.14$ days. The severity of anti-TB DIH is milder for early detection patient. TB patient with antiTB DIH received hepatoprotective agents to improve the liver function. The process of reintroduced hepatotoxic anti-TB drugs started after liver function test, and clinical symptoms are improved. The risk of unsuccessful outcome TB 2.5-fold higher for TB patient with anti-TB DIH compares to TB patient without anti-TB DIH. Most of the TB patient with anti-TB DIH had prolonged duration of TB treatment to reach the successful outcome. In summary, the anti-TB DIH had an impact on TB treatment.

\section{ACKNOWLEDGMENTS}

The authors would like to say thank you to all staff medical record department and TB-DOTS team at the hospital that provided source documents for this research.

\section{REFERENCES}

1. Zumla A, Raviglione M, Hafner R, von Reyn CF. Tuberculosis. N Eng J Med 2013;368(8):745-55.

2. World Health Organization. Global Tuberculosis Report 2015. Geneva: World Health Organization; 2016.

3. Singla R, Sharma SK, Mohan A, Makharia G, Sreenivas V, Jha B, et al. Evaluation of risk factors for anti-tuberculosis treatment induced hepatotoxicity. Indian J Med Res 2010;132:81-6.

4. Tostmann A, Boeree MJ, Aarnoutse RE, de Lange WC, van der Ven AJ, Dekhuijzen R. Anti-tuberculosis drug-induced hepatotoxicity: Concise 
up-to-date review. J Gastroenterol Hepatol 2008;23(2):192-202.

5. Verma R, Mahor GR, Shirvastava AK, Pathak P. Adverse drug reactions with first-line anti-tubercular drugs in a tertiary care hospital of central India: A study of clinical presentations, causality, and severity. Asian J Pharm Clin Res 2014;7(5):140-3

6. Siddiqui S, Baig MM, Jafer S, Ansari SF. Study on prevalence of adverse drug reactions in patients suffering from tuberculosis in a tertiary care hospital. Int J Pharm Pharm Sci 2016;8(8):375-7.

7. Satyaraddi A, Velpandian T, Sharma SK, Vishnubhatla S, Sharma A, Sirohiwal A, et al. Correlation of plasma anti-tuberculosis drug levels with subsequent development of hepatotoxicity. Int J Tuberc Lung Dis 2014;18(2):188-95, i-iii.

8. Penghui S, Xia Y, Liu F, Wang X, Yuan Y, Hu D, et al. Incidence, clinical features and impact on anti-tuberculosis treatment of anti-tuberculosis induced liver injury (ATLI) in China. PLoS One 2011;6:7.

9. Ministry of Health of Indonesia. National Guideline for Medical Treatment of Tuberculosis. Jakarta: Ministry of Health of Indonesia; 2013.

10. An $\mathrm{H}, \mathrm{Wu} \mathrm{X}$, Wang $\mathrm{Z}, \mathrm{Xu}$ J, Zheng $\mathrm{S}$, Wang $\mathrm{K}$. The clinical characteristics of anti-tuberculosis drug induced liver injury in 2457 hospitalized patients with tuberculosis in China. Afr J Pharm Pharmacol 2013;7(13):710-4.

11. World Health Organization. Treatment of Tuberculosis Guidelines. $4^{\text {th }}$ ed. Geneva: World Health Organization; 2010.

12. Sharma SK. Tuberculosis. $2^{\text {nd }}$ ed. New Delhi: Jaypee Brothers Medical Publisher (P) Ltd.; 2009.

13. Sharifzadeh M, Rasoulinejad M, Valipour F, Nouraie M, Vaziri S. Evaluation of patient-related factors associated with causality, preventability, predictability and severity of hepatotoxicity during antituberculosis [correction of anti-tuberclosis] treatment. Pharmacol Res 2005;51(4):353-8

14. Abera W, Cheneke W, Abebe G. Incidence of anti-tuberculosis-druginduced hepatotoxicity and associated risk factors among tuberculosis patients in Dawro Zone, South Ethiopia: A cohort study. Int J
Mycobacteriol 2016;5(1):14-20.

15. Buntoro IK, Kristin E, Sumardi S. Decrease of liver function after treatment of anti-tuberculosis drugs in tuberculosis patients with malnutrition and alcohol consumption. Int $\mathrm{J}$ Pharm Pharm Sci 2016;8(7):269-73

16. Chemotherapy and management of tuberculosis in the United Kingdom: Recommendations 1998. Joint Tuberculosis Committee of the British Thoracic Society. Thorax 1998;53:536-48.

17. Saukkonen JJ, Cohn DL, Jasmer RM, Schenker S, Jereb JA, Nolan CM, et al. An official ATS statement: Hepatotoxicity of anti-tuberculosis therapy. Am J Respir Crit Care Med 2006;174(8):935-52

18. Sharma SK, Singla R, Sarda P, Mohan A, Makharia G, Jayaswal A, et al. Safety of 3 different reintroduction regimens of anti-tuberculosis drugs after development of anti-tuberculosis treatment-induced hepatotoxicity. Clin Infect Dis 2010;50(6):833-9.

19. Devarbhavi H. Anti-tuberculous drug-induced liver injury: Current perspective. Trop Gastroenterol 2011;32(3):167-74.

20. Agal S, Baijal R, Pramanik S, Patel N, Gupte P, Kamani P, et al. Monitoring and management of anti-tuberculosis drug induced hepatotoxicity. J Gastroenterol Hepatol 2005;20:1745-52.

21. Wu S, Xia Y, Lv X, Zhang Y, Tang S, Yang Z, et al. Effect of scheduled monitoring of liver function during anti-tuberculosis treatment in a retrospective cohort in China. BMC Public Health 2012;12(11):454

22. Muñoz-Sellart M, Cuevas LE, Tumato M, Merid Y, Yassin MA. Factors associated with poor tuberculosis treatment outcome in the Southern region of Ethiopia. Int J Tuberc Lung Dis 2010;14(8):973-9.

23. Baker MA, Harries AD, Jeon CY, Hart JE, Kapur A, Lönnroth K, et al. The impact of diabetes on tuberculosis treatment outcomes: A systematic review. BMC Med 2011;9:81.

24. Singanayagam A, Sridhar S, Dhariwal J, Abdel-Aziz D, Munro K, Connell DW, et al. A comparison between two strategies for monitoring hepatic function during anti-tuberculous therapy. Am J Respir Crit Care Med 2012;185(6):653-9. 